\title{
Editorial
}

\section{Quantum Gravitational Spectroscopy}

\author{
Valery V. Nesvizhevsky, ${ }^{1}$ Ignatios Antoniadis, ${ }^{2}$ Stefan Baessler, ${ }^{3,4}$ and Guillaume Pignol ${ }^{5}$ \\ ${ }^{1}$ Institut Max von Laue-Paul Langevin, 38042 Grenoble, France \\ ${ }^{2}$ The European Organization for Nuclear Research, 1211 Geneva, Switzerland \\ ${ }^{3}$ Physics Department, University of Virginia, Charlottesville, VA 22904, USA \\ ${ }^{4}$ Oak Ridge National Laboratory, Oak Ridge, TN 37831, USA \\ ${ }^{5}$ Laboratoire de Physique Subatomique et de Cosmologie/IN2P3-UJF-INPG, 38000 Grenoble, France
}

Correspondence should be addressed to Valery V. Nesvizhevsky; nesvizhevsky@ill.eu

Received 31 December 2014; Accepted 31 December 2014

Copyright (C) 2015 Valery V. Nesvizhevsky et al. This is an open access article distributed under the Creative Commons Attribution License, which permits unrestricted use, distribution, and reproduction in any medium, provided the original work is properly cited. The publication of this article was funded by SCOAP S $^{3}$

Quantum gravitational spectroscopy with ultracold systems is an emerging field of particle physics based on recent major advances in related experimental and theoretical developments. The gravitational spectroscopy is profiting from its exceptional sensitivity due to extreme weakness of the gravitational interaction compared to other fundamental interactions; thus it provides us with access to the precision frontier in particle physics and other domains. Quantum gravitational spectroscopy is the ultimate limit of gravitational spectroscopy, which studies/measures fragile and thus sensitive quantum states of ultracold particles and systems. To provide observable phenomena with ultracold systems, like neutrons, atoms, or antiatoms, in gravitational quantum states, one has to produce them with sufficiently high phase-space density. Some of such studies in a gravitational field, like those with ultracold neutrons, have become reality; others with ultracold atoms and antiatoms are in preparation.

This subject is traditionally covered in a series of GRANIT Workshops (http://lpsc.in2p3.fr/Indico/conferenceDisplay .py?confld=947, https://lpsc.in2p3.fr/Indico/conferenceDisplay.py?confld=371, and http://lpsc.in2p3.fr/congres/granit06/index.php) [1]. Quantum gravitational spectroscopy with ultracold systems plays an important interdisciplinary role on a crossroad of many scientific domains. We are particularly interested in contributions describing research to be done with the new GRANIT facility, including not only all aspects from the theoretical motivation and analysis of already measured results but also potentially new areas, methodical and instrumental developments, which could improve its performance and universality, and finally analyzing priorities of these studies.

The topics include the following: (i) the GRANIT facility, measurements of gravitational quantum states of neutrons, including experimental results and theoretical developments, and also neutron interferometry in broad sense; (ii) novel instruments and methods for gravitational spectroscopy and interferometry, including those capable of improving the performance of GRANIT facility; (iii) fundamental interactions in near-surface quantum systems, including shortrange forces, chameleon-like forces, noncommutative quantum mechanics, emerging gravity, and antimatter and gravity; (iv) precision fundamental measurements with ultracold systems, including gravity with atoms, molecules, neutrons, and antimatter, quantum reflection, and dissipation; (v) surface studies with the GRANIT facility, including materials with predefined properties, levitating nanoparticles, and whispering gallery effect.

Bound states of a system could be formed by interactions of different kind. Detailed studies of such states do not only serve to explore the system itself, but also are a good tool to better understand the interaction responsible for the binding force. The problem is that a gravitational force is very weak to form easily a quantum bound system. One could cite Brian Hatfield in "Feynman Lectures on Gravitation" published in 1995 by Addison-Wesley Publishing Company: "Let us consider another possibility, an atom held together by gravity alone. For example, we might have two neutrons in a bound state. When we calculate the Bohr radius of such an atom, we 
find that it would be $10^{8}$ light years, and that the atomic binding energy would be $10^{-70}$ Rydbergs. There is then little hope of ever observing gravitational effects on systems which are simple enough to be calculable in quantum mechanics." However, gravitationally bound quantum states of neutrons were discovered in a quite special configuration of the potential.

For this observation, one used the fact that ultracold neutrons (UCNs) experience a neutron-optical potential (sometimes called Fermi potential) in matter of a size big enough that matter can totally reflect UCNs if their energy is sufficiently low. In fact, the term "ultracold neutron" is loosely defined as the neutrons which are totally reflected at all incidence angles by the matter used for the confining bottle for them. Here, we focus on UCNs with even lower energy, for which we can approximate the potential in matter as infinite. These neutrons bounce on a horizontal table essentially like ping-pong balls on a table. They are confined in a box formed by the infinite potential of the table and the linear gravitational potential. In order to detect effects of the quantization, one has to focus on neutrons in one of the lowest possible states in such a system.

General solutions of the corresponding one-dimensional Schrödinger equation are given in all major textbooks on quantum mechanics, for instance, in $[2,3]$. In relation to the neutron, this problem was considered for the first time in [4]. Energies of the lowest quantum states are very small, a few $\mathrm{peV}$, which can be compared with the upper energy limit for UCNs which is of the order of hundred neV. The size of the lowest quantum states is of the order of tens of micrometers.

The aim of previous experiments performed in the last decade was to discover these quantum states. For the discovery, a method was used that is essentially a measurement of the height and shape of wave functions [5-7]. The accuracy of such height measurement was not very high, $\sim 10^{-1}$. In order to improve it, efforts are currently undertaken by qBounce and Tokyo collaborations; the methods used are resonant transitions between quantum states induced by mechanical vibrations of the bottom mirror [8] and precision studies of wave functions of neutrons in gravitational quantum states [9]. Analysis of systematic effects and estimations of actual accuracy are in progress. The GRANIT collaboration is aiming at achieving an ultimate accuracy using long storage of UCNs in gravitational quantum states in a closed trap [10] with a dedicated UCN source [11] with high phase-space density to be put in reliable operation soon.

One of the main goals for improving the accuracy of quantum gravitational spectroscopy with neutrons is searches for extra short-range fundamental forces [12]. As usual, we discuss also any progress in all competing nonneutron methods as well as constraints at other characteristic distances. Among major methodical developments related to the phenomenon of gravitational quantum states are the detailed theoretical analysis and the planning experiments on observation of gravitational quantum states of antihydrogen atoms.

\section{Acknowledgments}

Over 50 participants from 12 countries in 4 continents attended the previous GRANIT-2014 Workshop. The organizers would like to thank ILL, IN2P3/CNRS, and UJF/Grenoble for financial support of the Workshop as well as for their help in preparation of the Workshop.

\author{
Valery V. Nesvizhevsky \\ Ignatios Antoniadis \\ Stefan Baessler \\ Guillaume Pignol
}

\section{References}

[1] I. Antoniadis, S. Baessler, O. Bertolami et al., "Workshop GRANIT-2010, 14-19 February 2010, Les Houches, France," Comptes Rendus Physique, vol. 12, no. 8, pp. 703-706, 2011.

[2] I. I. Goldman, V. D. Krivchenkov, V. I. Kogan, and V. M. Galitski, Problems in Quantum Mechanics, Academic Press, New York, NY, USA, 1960.

[3] J. J. Sakurai, Modern Quantum Mechanics, Benjamin/Cummings, Menlo Park, Calif, USA, 1985.

[4] V. I. Luschikov and A. Frank, "Quantum effects occuring when ultracold neutrons are stored on a plane," JETP Letters, vol. 28, pp. 559-561, 1978.

[5] V. V. Nesvizhevsky, H. G. Börner, A. K. Petukhov et al., "Quantum states of neutrons in the Earth's gravitational field," Nature, vol. 415, no. 6869, pp. 297-299, 2002.

[6] V. V. Nesvizhevsky, H. G. Börner, A. M. Gagarski et al., "Measurement of quantum states of neutrons in the Earth's gravitational field," Physical Review D, vol. 67, Article ID 102002, 2003.

[7] V. V. Nesvizhevsky, A. K. Petukhov, H. G. Börner et al., "Study of the neutron quantum states in the gravity field," European Physical Journal C, vol. 40, no. 4, pp. 479-491, 2005.

[8] T. Jenke, P. Geltenbort, H. Lemmel, and H. Abele, "Realization of a gravity-resonance-spectroscopy technique," Nature Physics, vol. 7, no. 6, pp. 468-472, 2011.

[9] G. Ichikawa, S. Komamiya, Y. Kamiya et al., "Observation of the spatial distribution of gravitationally bound quantum states of ultracold neutrons and its derivation using the Wigner function," Physical Review Letters, vol. 112, no. 7, Article ID 071101, 2014.

[10] S. Baessler, M. Beau, M. Kreuz et al., "The GRANIT spectrometer," Comptes Rendus Physique, vol. 12, pp. 707-728, 2011.

[11] P. Schmidt-Wellenburg, K. H. Andersen, P. Courtois et al., "Ultracold-neutron infrastructure for the gravitational spectrometer GRANIT," Nuclear Instruments and Methods in Physics Research, Section A: Accelerators, Spectrometers, Detectors and Associated Equipment, vol. 611, no. 2-3, pp. 267-271, 2009.

[12] I. Antoniadis, S. Baessler, M. Büchner et al., "Short-range fundamental forces," Comptes Rendus Physique, vol. 12, no. 8, pp. 755-778, 2011. 

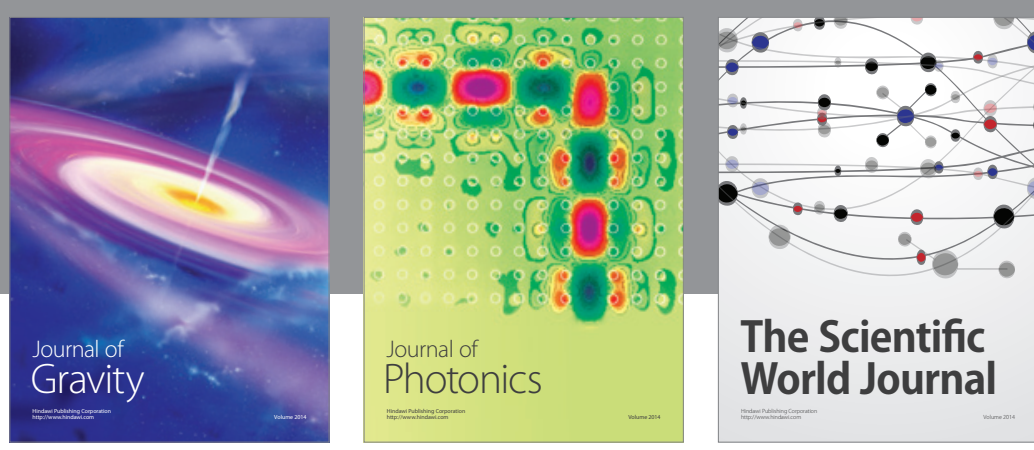

The Scientific World Journal
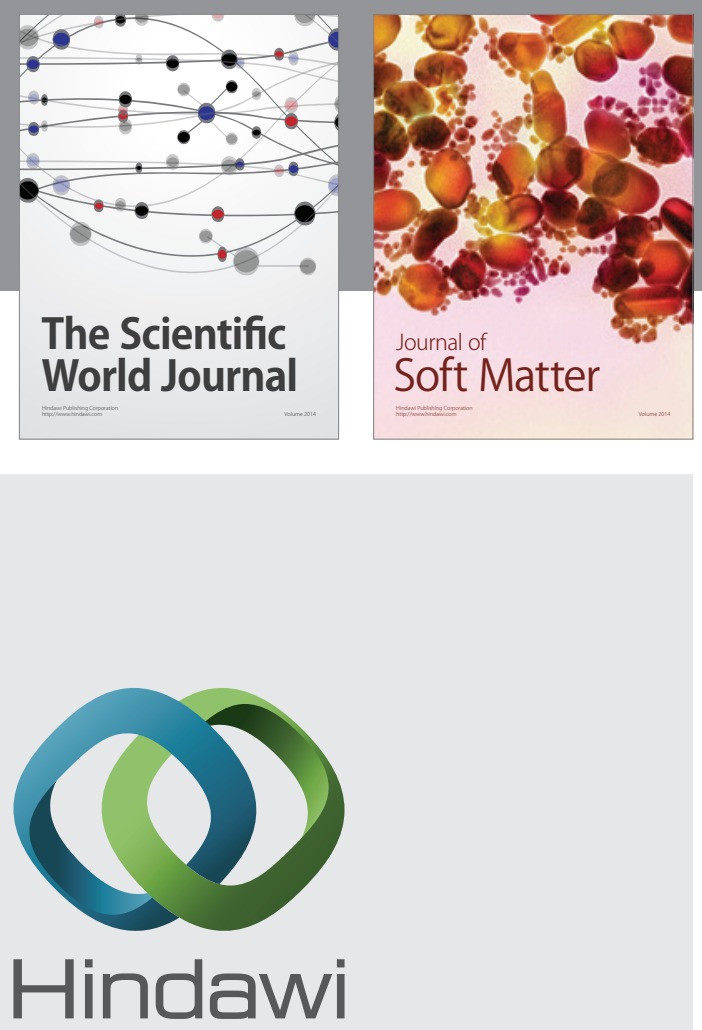

Submit your manuscripts at

http://www.hindawi.com

nternational Journal of

Statistical Mechanics
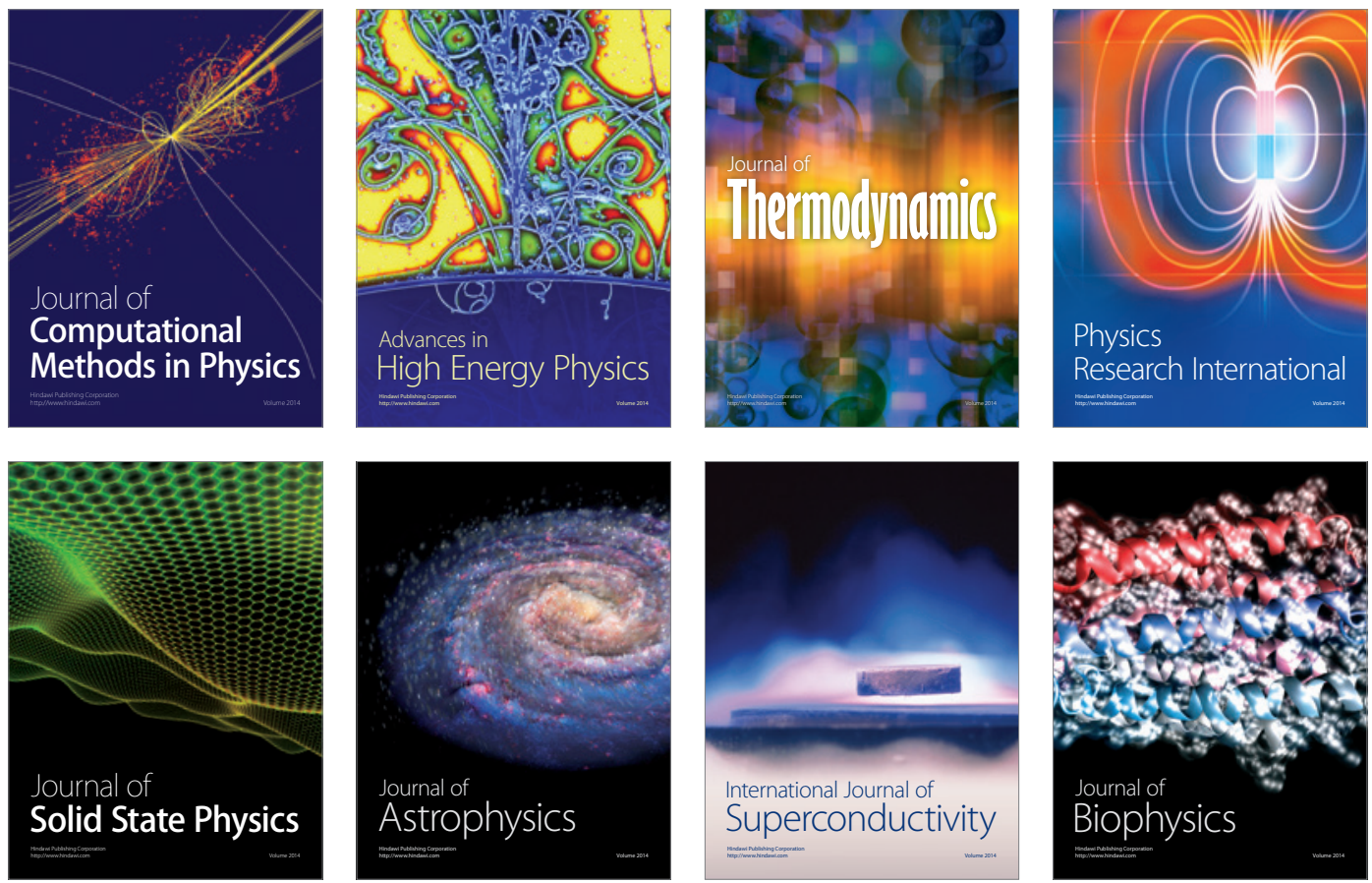
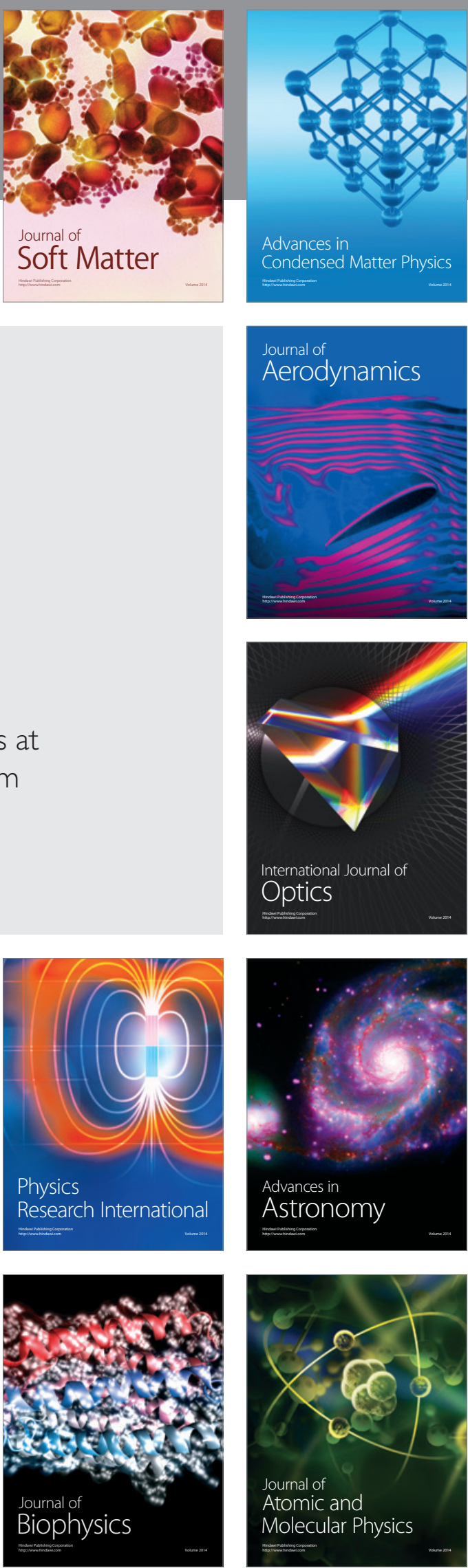